\title{
Palladium Nanoparticles Supported on Phosphorus-Doped Graphitic Carbon Nitride/Carbon Black Hybrids for Formic Acid Electro-Oxidation
}

\author{
Duo Yu ${ }^{1,2}$, Chenxi Lu ${ }^{1,2}$, Zhiguo Yan ${ }^{1,2}$, Xiaojun Yang ${ }^{1,2}$, Wei Yang ${ }^{1,4, *}$ Zaosheng Lv $^{3}$, \\ Zhengfang Tian ${ }^{5, *}$ and Qifeng Tian ${ }^{1,2, *}$ \\ ${ }^{1}$ Key Laboratory of Green Chemical Process of Ministry of Education \& Hubei Key Laboratory of \\ Novel Reactor and Green Chemical Technology (Wuhan Institute of Technology), Wuhan 430205, \\ People's Republic of China \\ ${ }^{2}$ School of Chemical Engineering and Pharmacy, Wuhan Institute of Technology, Wuhan 430205, \\ People's Republic of China \\ ${ }^{3}$ Key Laboratory of Hubei Province for Coal Conversion and New Carbon Materials, Wuhan \\ University of Science and Technology, Wuhan 430081, People's Republic of China \\ ${ }^{4}$ School of Chemistry and Environmental Engineering, Wuhan Institute of Technology, Wuhan \\ 430205, People's Republic of China \\ ${ }^{5}$ College of Chemistry and Chemical Engineering, Hubei Key Laboratory for Processing and \\ Application of Catalytic Materials, Huanggang Normal University, Huanggang 438000, People's \\ Republic of China \\ *E-mail: qftian@wit.edu.cn (Q. T. ), yangweight@126.com (W. Y. ), tzf7801@163.com (Z. T.)
}

Received: 6 July 2021 / Accepted: 14 August 2021 / Published: 10 September 2021

\begin{abstract}
A novel hybrid supports consisting of phosphorus-doped graphitic carbon nitride and carbon black were prepared for loading Pd nanoparticles (NPs). The obtained Pd/C-PCN catalysts exhibited superior catalytic performance towards formic acid electro-oxidation (FAO). The anchoring effect of the graphite carbon nitride $\left(\mathrm{g}-\mathrm{C}_{3} \mathrm{~N}_{4}\right)$ improved the dispersibility of Pd NPs. It is found that the Pd NPs with a reduced average diameter dispersed on $\mathrm{g}-\mathrm{C}_{3} \mathrm{~N}_{4}$ supports compared to that on carbon black. After doping with phosphorus, the prepared $\mathrm{Pd} / \mathrm{C}-\mathrm{PCN}-\mathrm{X}$ catalysts has a further improvement in the activity and durability of FAO. Pd/C-PCN-0.2 shows the highest peak current density for FAO. Its peak current density reached $771 \mathrm{~A} / \mathrm{g}$, which is 2.15 times that of $\mathrm{Pd} / \mathrm{C}(358 \mathrm{~A} / \mathrm{g})$. It also exhibited the best stability for FAO. The enhanced catalytic performances of this Pd/C-PCN-X catalysts can be attributed to high dispersion of Pd NPs and improved electronic structure of Pd NPs caused by P doping.
\end{abstract}

Keywords: carbon nitride; phosphorus-doped; palladium; formic acid oxidation; electrocatalysis $\underline{\text { FULL TEXT }}$ 
(C) 2021 The Authors. Published by ESG (www.electrochemsci.org). This article is an open access article distributed under the terms and conditions of the Creative Commons Attribution license (http://creativecommons.org/licenses/by/4.0/). 